OPEN ACCESS

Edited by:

Shihui Yang,

Hubei University, China

Reviewed by: Yong $\mathrm{Xu}$,

Nanjing Forestry University, China Zhiqiang Wen,

Nanjing University of Science and Technology, China

*Correspondence:

Sachin Kumar

sachin.biotech@gmail.com

tPresent address: Richa Arora

Department of Microbiology, School of Bioengineering and Biosciences, Lovely Professional University, Phagwara, India

Specialty section: This article was submitted to Bioenergy and Biofuels, a section of the journal Frontiers in Energy Research

Received: 20 September 2017 Accepted: 27 November 2017 Published: 12 December 2017

Citation:

Sharma NK, Behera S, Arora R and Kumar S (2017) Evolutionary Adaptation of Kluyveromyces marxianus NIRE-K3 for Enhanced Xylose Utilization.

Front. Energy Res. 5:32. doi: 10.3389/fenrg.2017.00032

\section{Evolutionary Adaptation of Kluyveromyces marxianus NIRE-K3 for Enhanced Xylose Utilization}

\author{
Nilesh Kumar Sharma ${ }^{1,2}$, Shuvashish Behera ${ }^{1}$, Richa Arora ${ }^{1 \dagger}$ and Sachin Kumar ${ }^{1 *}$ \\ ${ }^{1}$ Biochemical Conversion Division, Sardar Swaran Singh National Institute of Bio-Energy, Kapurthala, India, \\ I. K. Gujral Punjab Technical University, Kapurthala, India
}

The evolutionary adaptation was approached on the thermotolerant yeast Kluyveromyces marxianus NIRE-K3 at $45^{\circ} \mathrm{C}$ on xylose as a sole source of carbon for enhancement of xylose uptake. After 60 cycles, evolved strain K. marxianus NIRE-K3.1 showed comparatively 3.75- and 3.0-fold higher specific growth and xylose uptake rates, respectively, than that of native strain. Moreover, the short lag phase was also observed on adapted strain. During batch fermentation with xylose concentration of $30 \mathrm{~g} \mathrm{l}^{-1}$, K. marxianus NIRE-K3.1 could utilize about $96 \%$ of xylose in $72 \mathrm{~h}$ and produced 4.67 and $15.7 \mathrm{~g} \mathrm{l}^{-1}$ of ethanol and xylitol, respectively, which were 9.72- and 4.63-fold higher than that of native strain. Similarly, specific sugar consumption rate, xylitol, and ethanol yields were 5.07-, 1.15-, and 2.44-fold higher as compared to the native strain, respectively. The results obtained after evolutionary adaptation of $K$. marxianus NIRE-K3 show the significant improvement in the xylose utilization, ethanol and xylitol yields, and productivities. By understanding the results obtained, the significance of evolutionary adaptation has been rationalized, since the adapted culture could be more stable and could enhance the productivity.

Keywords: evolutionary adaptation, xylose, Kluyveromyces marxianus, ethanol, xylitol

\section{INTRODUCTION}

Several environmental issues including global warming, climate change, $\mathrm{CO}_{2}$, and $\mathrm{CO}$ emission are major concern nowadays, which are primarily caused by conventional transportation fuels and burning of the crop residues (Kumar et al., 2009). To cure these issues, a sustainable process should be developed to produce alternative transportation fuel along with utilization of crop residues. Lignocellulosic ethanol production could be a better solution to resolve these issues, which has already been proved as better alternative to conventional fuels including gasoline (Kumar et al., 2009, 2010; Chandel et al., 2011). Lignocellulosic waste has also been found as a better source for economic bioethanol production due to its abundance and low cost (Kumar et al., 2010; Sharma et al., 2016). It contains glucose, galactose, mannose, xylose, and arabinose in which glucose and xylose covers about $90 \%$ of total sugar present in biomass (Kumar et al., 2010; Sharma et al., 2016). Therefore, both these sugars needed to be fermented for economical process development. Conventional yeast Saccharomyces cerevisiae can only ferment glucose, but unable to ferment xylose due to lack of xylose metabolic genes (Kumar et al., 2010; Chandel and Singh, 2011; Kuhad et al., 2011; Sharma et al., 2016). 
Various attempts have also been made to establish xylose metabolic pathway into $S$. cerevisiae through genetic engineering by incorporating xylose reductase-xylitol dehydrogenase or xylose isomerase pathway for the coutilization of glucose and xylose (Kuyper et al., 2005; Liu and Hu, 2010; Zhou et al., 2012). However, there are various limitations including competitive inhibition due to presence of glucose and degradation of xylose transporter, which inhibits coutilization of xylose (Apel et al., 2016). On the other hand, numerous mesophilic xylose-utilizing yeast strains have been tested to ferment xylose, but out of them only few including Scheffersomyces stipitis, Candida shehatae, C. intermedia, and Debaryomyces hansenii showed significant ethanol fermentation ability on xylose (Kuhad et al., 2011; Sharma et al., 2014). However, there were various hurdles including low ethanol productivity, low sugar and ethanol tolerance, and slow xylose uptake rate (Kuhad et al., 2011; Sharma et al., 2014). Furthermore, mesophiles also have limitations like low productivity in simultaneous saccharification and fermentation (SSF) at low temperatures, energy requirements for mixing and product recovery, which increases the process cost (Kumar et al., 2010; Zhang et al., 2013). Besides, a thermotolerant yeast have various advantages over mesophiles including higher rate of saccharification and fermentation, utilization of broad range of substrates and less energy requirement for mixing and product recovery, and lower cost of pumping and steering (Kumar et al., 2010; Rodrussamee et al., 2011; Zhang et al., 2013; Sharma et al., 2016). Nowadays, Kluyveromyces marxianus getting more attention due to thermotolerance, broad substrate utilization, and higher growth rate (Wilkins et al., 2008; Zhang et al., 2013; Sharma et al., 2016). It can grow and produce ethanol up to $50^{\circ} \mathrm{C}$ (Kumar et al., 2010). Furthermore, SSF is a major application using this strain, which takes place at higher temperature due to the maximum cellulases activity at $50^{\circ} \mathrm{C}$ (Arora et al., 2015). Moreover, requirements of heating and cooling process during saccharification and fermentation using mesophiles increases the cost of process (Kumar et al., 2009). K. marxianus shows great potential over mesophiles including no cooling requirement during SSF, solvent tolerance, and minimum risk of contamination, which proves it to be a better alternative for sustainable process development (Kumar et al., 2009; Arora et al., 2017).

Evolutionary adaptation has been employed to enhance growth and fermentation ability of strains along with tolerance to various inhibitors including hydroxymethylfurfural (HMF), furfural, and acetic acid (Agbogbo et al., 2008; Zhu et al., 2009). Evolutionary adaptation together with genetic engineering can be a better approach to develop the sustainable and economic process (Behera et al., 2016; Kuyper et al., 2005; Liu and Hu, 2010; Zhou et al., 2012; Sharma et al., 2016). This strategy has already been implemented along with various conventional techniques including protein engineering, intergeneric hybridization, and metabolic engineering (Kuhad et al., 2011; Zhang et al., 2015). Furthermore, evolutionary adaptation has been proven a useful approach for the development of sustainable technology (Kuyper et al., 2005; Liu and Hu, 2010; Zhou et al., 2012; Pereira et al., 2015).

In this study, evolutionary adaptation of $K$. marxianus NIRE-K3 was performed for xylose utilization and comparative assessment of native and adapted $K$. marxianus NIRE-K3 on the basis of growth, sugar uptake rate, product yield, and productivity on xylose as carbon source has been reported. The comparative kinetics study is also reported.

\section{MATERIALS AND METHODS}

\section{Microorganism and Growth Conditions}

Thermotolerant yeast K. marxianus NIRE-K3 (MTCC 5934) was used for evolutionary adaptation study. The yeast culture was grown at $45^{\circ} \mathrm{C}$ and maintained on yeast extract-peptone (YEPX) medium $\left[\left(\mathrm{gl}^{-1}\right)\right.$ : yeast extract (Himedia, Mumbai, India), 10; peptone (Himedia, Mumbai, India), 20; xylose (Himedia, Mumbai, India), 20; $\mathrm{pH}, 5.5]$. Growth was performed for $24 \mathrm{~h}$ at $45^{\circ} \mathrm{C}$ and $150 \mathrm{rpm}$ on an orbital shaker incubator (New Brunswick Innova 43/43R Shaker, Germany). The samples were collected at an interval of $2 \mathrm{~h}$ and analyzed for dry cell weight (DCW), xylose, glucose, and extracellular metabolites concentrations. Yeast cultures were maintained at $-80^{\circ} \mathrm{C}$ in stock with $30 \%$ glycerol (v/v). Similarly, evolved strain $K$. marxianus NIRE-K3.1 was also grown and maintained as same.

\section{Evolutionary Adaptation}

Evolutionary adaptation was performed in $100 \mathrm{ml}$ of cotton plugged Erlenmeyer flask containing 20-ml YEPX medium with $2 \%$ xylose as carbon source through sequential transfer of culture. The initial batch was performed by culturing loopful cells from phytagel plate in Erlenmeyer flask and incubated for $24 \mathrm{~h}$ at $45^{\circ} \mathrm{C}$ in an orbital shaker incubator (New Brunswick Innova 43/43R Shaker, Germany) at $150 \mathrm{rpm}$. Furthermore, 1\% inoculum was used for continual batch for subsequent growth. One milliliter sample was withdrawn after every $24 \mathrm{~h}$ for analysis of residual xylose, DCW, and metabolites produced during the growth. The adaptation was performed until no further improvement was observed in xylose utilization.

\section{Fermentation Conditions}

Fermentation was performed to check out the improvements in xylose utilization and fermentation capability of $K$. marxianus NIRE-K3.1 as compared to native strain. Inoculums for fermentation using $K$. marxianus NIRE-K3 and NIRE-K3.1 were prepared using YEPX medium with similar composition as that of growth medium. Fermentation was performed in $11 \mathrm{screw-}$ caped Erlenmeyer flask with working volume of $200 \mathrm{ml}$ of YEPX medium containing $30 \mathrm{~g} \mathrm{l}^{-1}$ xylose at a temperature of $45^{\circ} \mathrm{C}$ and pH 5.5 with agitation of $150 \mathrm{rpm}$ in an orbital shaker incubator. The initial cell mass concentration was kept at $2 \mathrm{~g} \mathrm{l}^{-1}$.

\section{Analytical Methods}

Samples collected during growth and fermentation were centrifuged using Eppendorf centrifuge 5430R at 5,000 $\mathrm{g}$ for $10 \mathrm{~min}$, followed by washing the pellet twice with deionized water and further drying in a vacuum oven at $80^{\circ} \mathrm{C}$ to a constant weight, followed by DCW analysis. The supernatant was used to analyze xylose, ethanol, xylitol, glycerol, and acetic acid using HPLC (Agilent Technologies) with HiPlex $\mathrm{H}$ column at $57^{\circ} \mathrm{C}$ with $1 \mathrm{mM} \mathrm{H}_{2} \mathrm{SO}_{4}$ as the mobile phase at a flow rate of $0.7 \mathrm{ml} \mathrm{min}^{-1}$ 
and detected by refractive index detector at $50^{\circ} \mathrm{C}$. All the experiments were carried out in triplicate, and the given values are the mean values.

\section{Kinetics}

Fermentation kinetic parameters were calculated according to Sharma et al. (2016):

$$
\mu=\text { Standardized value for specific }
$$
growth rate of K. marxianus NIRE-K3

$Y_{\mathrm{X} / \mathrm{S}}=\frac{\text { Mass of biomass (yeast cell) formed }}{\text { Mass of substrate (xylose) consumed }}$

$$
Y_{\mathrm{P} / \mathrm{S}}=\frac{\text { Mass of product formed }}{\text { Mass of substrate (xylose) consumed }}
$$

$Q_{S}=$ Substrate (xylose) uptake $(g)$ per liter per hour

$Q_{\mathrm{p}}=$ Product formed $(\mathrm{g})$ per liter per hour

$$
\begin{aligned}
& q_{\mathrm{s}}=\frac{\text { Mass of substrate (xylose) consumed }}{\text { Mass of biomass (yeast cell) formed }} \mu \\
& q_{\mathrm{p}}=\frac{\text { Mass of product formed }}{\text { Mass of biomass (yeast cell) formed }} \mu .
\end{aligned}
$$

\section{RESULTS AND DISCUSSION}

Potential of adapted strain K. marxianus NIRE-K3.1 for xylose uptake during growth and fermentation was compared with native strain.

\section{Adaptation of $K$. marxianus NIRE-K3}

Kluyveromyces marxianus NIRE-K3 was adapted for enhanced xylose utilization through sequential culturing in YEPX medium containing $20 \mathrm{~g} \mathrm{l}^{-1}$ xylose. The reliability of strategy was evaluated by monitoring improvement in growth and sugar assimilation after each batch. There was a linear improvement in xylose utilization rate (Figure 1). After 45 batches of subculturing, $82.28 \%$ xylose could be utilized in $24 \mathrm{~h}$ of initial sugar provided, i.e., $20 \mathrm{~g} \mathrm{l}^{-1}$ and produced $5.8 \mathrm{~g} \mathrm{l}^{-1}$ cell biomass as compared to $17.43 \%$ for native strain under same conditions. Furthermore, the improvement in xylose utilization was slow, which might be due to reprogramming of internal metabolic pathway through increased xylose utilization (New et al., 2014). Furthermore, subculturing was continued up to 60 batches to check the further improvement in xylose utilization. However, the improvement was not significant, and no further subculturing was done. There was $86.28 \%$ of xylose utilization after 60 batches with the formation of $5.9 \mathrm{~g} \mathrm{l}^{-1}$ cell biomass, which was 4.9- and 4.21-fold higher than that of native strain, respectively (Figure 1). After 60 batches, the adapted strain was renamed as K. marxianus NIRE-K3.1. In another study, xylose assimilation could be enhanced 2.81-fold through evolutionary adaptation of $K$. marxianus NIRE-K1 (Sharma et al., 2016). Qi et al. (2015) reported the evolutionary adaptation for reprogramming pre- and post-transcriptional pathway for less genetically explored microorganisms. Kahr et al. (2011) reported enhanced ethanol production using adapted

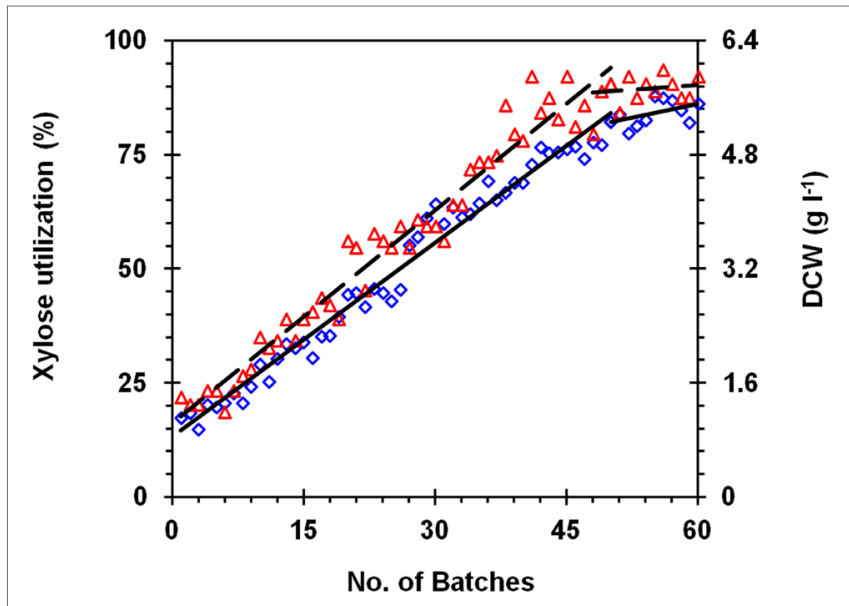

FIGURE 1 | Adaptation of Kluyveromyces marxianus NIRE-K3 in yeast extract-peptone media. $(\diamond)$ xylose utilization; $(\Delta)$ dry cell weight (DCW); $(-)$ linear xylose utilization; (- $)$ linear DCW.

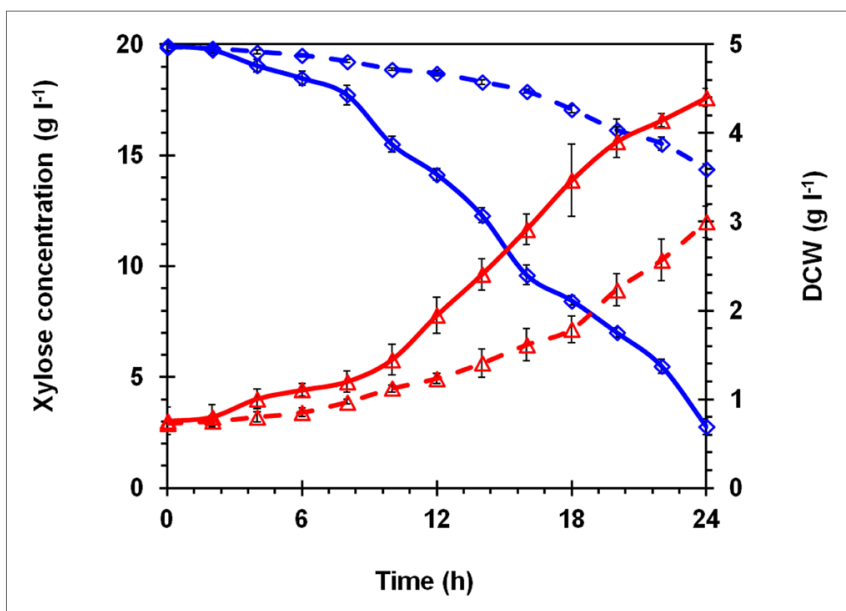

FIGURE 2 | Comparison of growth pattern between native Kluyveromyces marxianus NIRE-K3 (- ) and K. marxianus NIRE-K3.1 (adapted up to 60 batches) (-) using xylose as a carbon source; $(\diamond)$ xylose; $(\Delta)$ dry cell weight (DCW).

S. stipitis on xylose, which could be increased up to $4.6 \%$ vol from 3.4\% vol. In another study, Garcia Sanchez et al. (2010) reported the enhanced xylose and arabinose utilization in recombinant $S$. cerevisiae TMB3130 through evolutionary adaptation by chemostat aerobic adaptation of about 1800 hours.

\section{Comparative Growth Analysis of $K$. marxianus NIRE-K3 and NIRE-K3.1}

To ensure the improvement in K. marxianus NIRE-K3.1, comparative growth profiling was carried out and compared with native $K$. marxianus NIRE-K3. Both the strains were cultivated in 500-ml cotton plugged Erlenmeyer flasks with working volume of $100 \mathrm{ml}$ in YEP medium containing $20 \mathrm{~g} \mathrm{l}^{-1}$ xylose for $24 \mathrm{~h}$. Figure 2 shows the growth pattern of $K$. marxianus NIRE-K3 and 
TABLE 1 | Growth kinetics of Kluyveromyces marxianus NIRE-K3 and NIRE-K3.1.

\begin{tabular}{|c|c|c|c|c|}
\hline \multirow{2}{*}{$\begin{array}{l}\text { Kinetic parameters } \\
\text { Growth }\end{array}$} & \multicolumn{2}{|c|}{ K. marxianus NIRE-K3 } & \multicolumn{2}{|c|}{ K. marxianus NIRE-K3.1 } \\
\hline & Glucose & Xylose & Glucose & Xylose \\
\hline Maximum specific growth rate $\left(\mu_{\max }, \mathrm{h}^{-1}\right)$ & $0.29 \pm 0.01$ & $0.02 \pm 0.003$ & $0.31 \pm 0.01$ & $0.075 \pm 0.01$ \\
\hline Ethanol yield $\left(Y_{p / s}, g^{-1}\right)$ & $0.35 \pm 0.007$ & $0.009 \pm 0.001$ & $0.41 \pm 0.002$ & $0.06 \pm 0.001$ \\
\hline Xylitol yield $\left(Y_{p / s}, g^{-1}\right)$ & - & $0.07 \pm 0.009$ & - & $0.17 \pm 0.01$ \\
\hline Cell yield $\left(Y_{x / s}, g^{-1}\right)$ & $0.26 \pm 0.005$ & $0.43 \pm 0.06$ & $0.27 \pm 0.002$ & $0.22 \pm 0.01$ \\
\hline Specific sugar consumption rate (xylose) $\left(q_{\mathrm{s}}, \mathrm{g} \mathrm{g}^{-1} \mathrm{~h}^{-1}\right)$ & & $0.13 \pm 0.01$ & & $0.38 \pm 0.02$ \\
\hline
\end{tabular}

NIRE-K3.1. About $5.35 \pm 0.23 \mathrm{~g} \mathrm{l}^{-1}$ xylose could be utilized by K. marxianus NIRE-K3 in $24 \mathrm{~h}$ of incubation, while K. marxianus NIRE-K3.1 could utilize $17.15 \pm 0.36 \mathrm{~g} \mathrm{l}^{-1}$ xylose. Shortened lag phase was also observed for K. marxianus NIRE-K3.1. Mihoub et al. (2003), Landaeta et al. (2013), and Jõers and Tenson (2016) also reported the shorter lag phase for adapted strains. Moreover, xylose utilization of $K$. marxianus NIRE-K3.1 was about 3.21fold higher as compare to K. marxianus NIRE-K3 (Figure 2). The maximum specific growth rate $\left(\mu_{\max }\right)$ of $K$. marxianus NIRE-K3.1 was calculated as $0.075 \pm 0.01 \mathrm{~h}^{-1}$ which was 3.75 -fold higher than K. marxianus NIRE-K3 $\left(0.02 \pm 0.003 \mathrm{~h}^{-1}\right)$ (Table 1). Liu and $\mathrm{Hu}$ (2010) developed a xylose-utilizing $S$. cerevisiae through combination of genetic engineering and adaptation, which showed improved xylose utilization with higher specific growth rate $\left(0.225 \mathrm{~h}^{-1}\right)$ than native recombinant strain $\left(0.055 \mathrm{~h}^{-1}\right)$ by using xylose as carbon source under aerobic environment. Similarly, S. cerevisiae TMB3400 was adapted for 97 batches in presence of cocktail of 12 different inhibitors and reported increased maximum specific growth rate of $0.33 \mathrm{~h}^{-1}$ along with shorter lag phase (Koppram et al., 2012).

The xylose uptake rate $\left(q_{\mathrm{s}}\right)$ for $K$. marxianus NIRE-K3.1 was also calculated as $0.38 \pm 0.02 \mathrm{~g} \mathrm{~g}^{-1} \mathrm{~h}^{-1}$, which was 3.0 -fold higher than that of $K$. marxianus NIRE-K3 $\left(0.13 \pm 0.01 \mathrm{~g} \mathrm{~g}^{-1} \mathrm{~h}^{-1}\right)$ (Table 1). Mohagheghi et al. (2014) reported the adaptation of Zymomonas mobilis $8 \mathrm{~b}$ in the presence of 2-deoxyglucose for coutilization of xylose and glucose present in pretreated corn strove. Evolved strain Z. mobilis \#7 could utilize $86 \%$ of xylose present in neutralized liquor as compared to parent strain (Mohagheghi et al., 2014). Similarly, Shen et al. (2012) applied adaptive evolution on recombinant $S$. cerevisiae BSPC095 for more than 1000 hours in xylose containing minimal media. Evolved strain S. cerevisiae BSPX013 showed 2.5- and 8.0-fold higher specific xylose consumption rate in glucose-xylose cofermentation and sole xylose fermentation, respectively (Shen et al., 2012). The ethanol yield was also reported to be increased from $0.37 \pm 0.02$ to $0.43 \pm 0.00 \mathrm{~g} \mathrm{~g}^{-1}$ using $S$. cerevisiae BSPX013. In another study, Candida guilliermondii was adapted on rice straw hemicellulosic hydrolyzate and reported the increased xylose consumption up to $83 \%$ in adapted strain (Silva and Roberto, 2001).

In this study, ethanol and xylitol yields $\left(Y_{\mathrm{p} / \mathrm{s}}, \mathrm{g} \mathrm{g}^{-1}\right)$ of NIRE-K3.1 were 6.67- and 2.42-fold higher than that of NIRE-K3, respectively. However, cell yield $\left(Y_{\mathrm{x} / \mathrm{s}}, \mathrm{g} \mathrm{g}^{-1}\right)$ of NIRE-K3.1 was $0.22 \pm 0.01 \mathrm{~g} \mathrm{~g}^{-1}$, whereas NIRE-K3 showed $0.43 \pm 0.06 \mathrm{~g} \mathrm{~g}^{-1}$ cell yield on xylose as sole carbon source (Table 1). The possible reason behind less cell yield of K. marxianus NIRE-K3.1 may be due to the product formation during growth period (Arora et al., 2015).

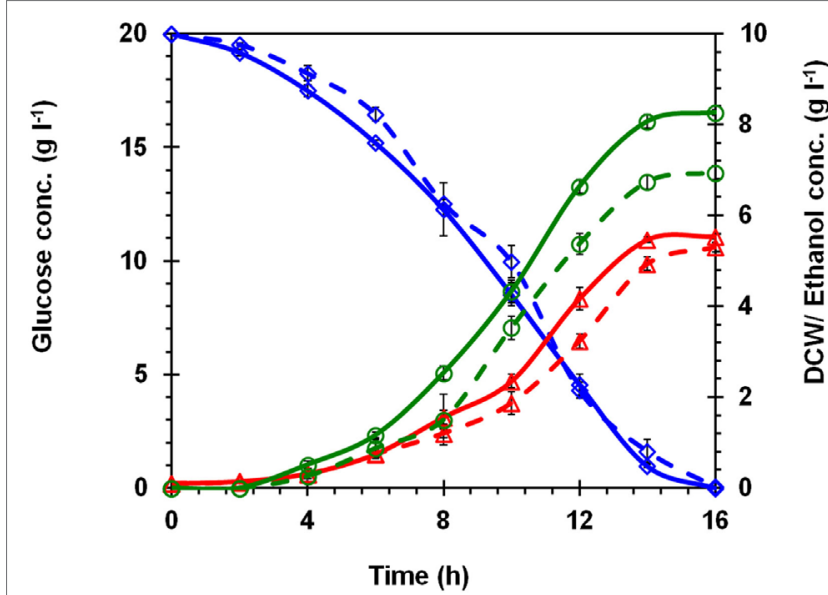

FIGURE 3 | Comparison of growth pattern between native Kluyveromyces marxianus NIRE-K3 (--) and K. marxianus NIRE-K3.1 (adapted up to 60 batches) (-); using glucose as a carbon source, $(\diamond)$ glucose; $(\Delta)$ dry cell weight; (o) ethanol.

The growth of $K$. marxianus NIRE-K3 and NIRE-K3.1 was also carried out in glucose containing medium (Figure 3). Both K. marxianus NIRE-K3 and NIRE-K3.1 showed the same lag phase and took $16 \mathrm{~h}$ to finish the glucose concentration of $20 \mathrm{~g} \mathrm{l}^{-1}$. Maximum specific growth rate $\left(\mu_{\max }\right)$ and cell yield $\left(Y_{\mathrm{x} / \mathrm{s}}\right)$ were also almost similar for both adaptive and native strains as shown in Table 1. However, the ethanol yield $\left(Y_{\mathrm{p} / \mathrm{s}}\right)$ using K. marxianus NIRE-K3.1 was 1.17-fold higher than that of $K$. marxianus NIRE-K3 (Table 1). This study shows no effect of evolutionary adaptation on the growth of K. marxianus NIRE-K3 and NIRE-K3.1, when grown on glucose as carbon source.

\section{Fermentation Profile of $K$. marxianus NIRE-K3 and NIRE-K3.1}

Fermentation was carried out in YEPX medium containing xylose concentration of $30 \mathrm{~g} \mathrm{l}^{-1}$ using $K$. marxianus NIRE-K3 and NIRE-K3.1 separately and drawn the xylose utilization and production of ethanol and xylitol pattern with time (Figure 4). During initial $12 \mathrm{~h}$ of fermentation, K. marxianus NIRE-K3.1 produced negligible amount of xylitol and ethanol, i.e., $0.014 \pm 0.002$ and $0.004 \pm 0.001 \mathrm{~g} \mathrm{l}^{-1}$, respectively, while there was no ethanol/xylitol was observed using $K$. marxianus NIRE-K3. After $24 \mathrm{~h}$ of fermentation, K. marxianus NIRE-K3.1 utilized $5.50 \pm 0.22$ and produced $1.008 \pm 0.108 \mathrm{~g} \mathrm{l}^{-1}$ xylitol, 


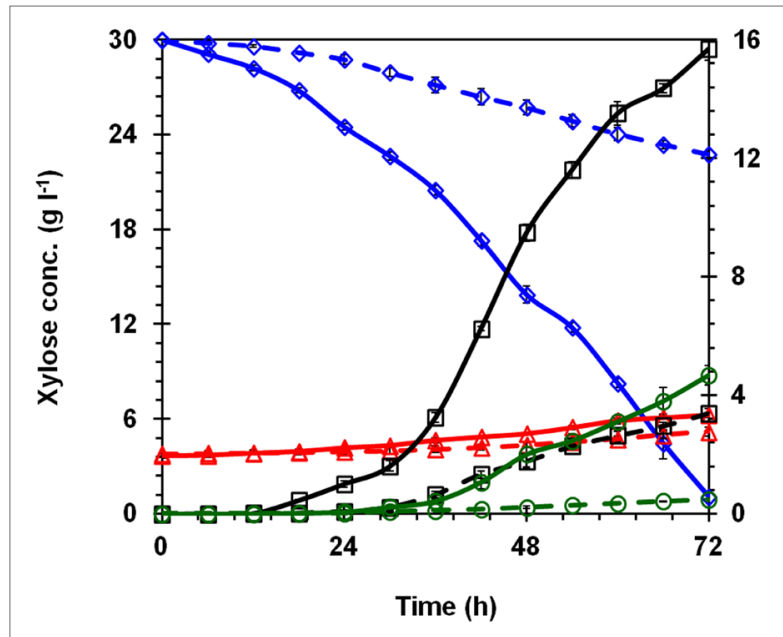

FIGURE 4 | Fermentation profile using optimized medium concentration in bench-scale bioreactor, native Kluyveromyces marxianus NIRE-K3 (- - ) and K. marxianus NIRE-K3.1 (adapted up to 60 batches) (-) using xylose as a carbon source; $(\diamond)$ xylose; $(\Delta)$ dry cell weight; (o) ethanol; $(\square)$ xylitol.
TABLE 2 | Fermentation kinetics of native and adapted Kluyveromyces marxianus NIRE-K3 and NIRE-K3.1.

\begin{tabular}{|c|c|c|}
\hline Kinetic parameters & $\begin{array}{l}\text { K. marxianus } \\
\text { NIRE-K3 }\end{array}$ & $\begin{array}{l}\text { K. marxianus } \\
\text { NIRE-K3.1 }\end{array}$ \\
\hline Ethanol $\left(P_{\text {ethanol }}, \mathrm{g}^{-1}\right)$ & $0.42 \pm 0.02$ & $4.67 \pm 0.33$ \\
\hline Xylitol $\left(P_{\text {xylitol }}, \mathrm{g} \mathrm{l}^{-1}\right)$ & $3.39 \pm 0.15$ & $15.7 \pm 0.4$ \\
\hline Cell yield $\left(Y_{x / s}, g^{-1}\right)$ & $0.10 \pm 0.015$ & $0.047 \pm 0.0019$ \\
\hline Ethanol yield $\left(Y_{p / s}, g^{-1}\right)$ & $0.066 \pm 0.0042$ & $0.16 \pm 0.01$ \\
\hline Xylitol yield $\left(Y_{p / s}, g^{-1}\right)$ & $0.46 \pm 0.035$ & $0.54 \pm 0.015$ \\
\hline Volumetric substrate uptake $\left(Q_{\mathrm{s}}, \mathrm{g} \mathrm{I}^{-1} \mathrm{~h}^{-1}\right)$ & $0.10 \pm 0.003$ & $0.40 \pm 0.002$ \\
\hline $\begin{array}{l}\text { Volumetric product productivity (ethanol) } \\
\left(Q_{p}, g^{\left.I^{-1} h^{-1}\right)}\right.\end{array}$ & $0.007 \pm 0.0003$ & $0.065 \pm 0.004$ \\
\hline $\begin{array}{l}\text { Volumetric product productivity (xylitol) } \\
\left(Q_{p}, \mathrm{~g} \mathrm{I}^{-1} \mathrm{~h}^{-1}\right)\end{array}$ & $0.047 \pm 0.002$ & $0.22 \pm 0.005$ \\
\hline $\begin{array}{l}\text { Specific sugar consumption rate (xylose) } \\
\left(q_{\mathrm{s}}, \mathrm{g} \mathrm{g}^{-1} \mathrm{~h}^{-1}\right)\end{array}$ & $0.012 \pm 0.001$ & $0.061 \pm 0.0014$ \\
\hline $\begin{array}{l}\text { Specific product formation rate (ethanol) } \\
\left(q_{p}, \mathrm{~g} \mathrm{~g}^{-1} \mathrm{~h}^{-1}\right)\end{array}$ & $0.0016 \pm 0.0003$ & $0.025 \pm 0.001$ \\
\hline $\begin{array}{l}\text { Specific product formation rate (xylitol) } \\
\left(q_{\mathrm{p}}, \mathrm{g} \mathrm{g}^{-1} \mathrm{~h}^{-1}\right)\end{array}$ & $0.011 \pm 0.001$ & $0.08 \pm 0.0016$ \\
\hline Conversion rate (\%) into ethanol & $13.23 \pm 0.83$ & $32.35 \pm 2.47$ \\
\hline Conversion rate (\%) into xylitol & $46.40 \pm 3.49$ & $53.75 \pm 1.51$ \\
\hline
\end{tabular}

lignocellulosic biomass (Liu and Hu, 2010; Kahr et al., 2011; Kuhad et al., 2011; Choudhary et al., 2017). Silva and Roberto (2001) adapted cells of C. guilliermondii for the fermentation on rice straw hemicellulosic hydrolyzate with and without nutrients and reported about 3.0-, 2.0-, and 1.9-fold increase in xylose utilization, xylitol yield, and productivity, respectively, as compared to unadapted cells after 120 hours. On another study, Shen et al. (2012) reported the enhanced ethanol yield of adapted strain of S. cerevisiae BSPX013, which increased up to $0.43 \pm 00 \mathrm{~g} \mathrm{~g}^{-1}$ from $0.37 \pm 0.02$ on xylose as a sole source of carbon, while xylitol and cell yield decreased.

Similarly, the cell yield of $K$. marxianus NIRE-K3.1 also decreased by $53 \%$ as compared to $K$. marxianus NIRE-K3 (Table 2). Decrease in cell yield of $K$. marxianus NIRE-K3.1 was due to conversion of maximum fraction of xylose into product formation rather than growth (Shen et al., 2012; Arora et al., 2015). Conversion rate of xylose into ethanol and xylitol in K. marxianus NIRE-K3.1 was $32.35 \pm 2.47$ and $53.75 \pm 1.51 \%$, which was 2.44- and 1.15-fold higher than that of $K$. marxianus NIRE-K3 $(13.23 \pm 0.83$ and $46.40 \pm 3.49 \%)$, respectively. Moreover, the specific product formation rate $\left(\mathrm{q}_{\mathrm{p}}, \mathrm{g} \mathrm{g}^{-1} \mathrm{~h}^{-1}\right)$ in $K$. marxianus NIRE-K3.1 was also found to be higher than that of unadapted culture (Table 2). The volumetric substrate uptake $\left(Q_{s}\right)$ and product formation rates $\left[\mathrm{Q}_{\mathrm{p}(\mathrm{xylitol})}, \mathrm{Q}_{\mathrm{p} \text { (ethanol) }}\right]$ were also calculated to be 4.0-, 4.62-, and 9.74-fold higher in adapted culture (Table 2).

The above results indicate the significant improvement in xylose utilization, ethanol and xylitol yields, and productivities using evolved strain of K. marxianus NIRE-K3. Furthermore, adaptation strategy also stabilizes heterologous gene expression in mutant strains (Diao et al., 2013; Lee et al., 2014). In the similar way, ethanol yield has also been augmented about twofold in recombinant $S$. cerevisiae after adaptation in bagasse hydrolyzate containing inhibitors including furaldehydes, phenolic compounds, and aliphatic acids (Martin et al., 2007). Moreover, several inhibiters tolerance including acetic acid, furfural, HMF, 
TABLE 3 | Comparison of xylose utilization and ethanol production between Kluyveromyces marxianus NIRE-K3.1 and reported yeast strains.

\begin{tabular}{|c|c|c|c|c|}
\hline Strain & Xylose utilization $\left(\mathrm{g} \mathrm{I}^{-1}\right)$ & Xylitol conc. ( $\left.\mathrm{g} \mathrm{I}^{-1}\right)$ & Ethanol conc. $\left(\mathrm{g} \mathrm{I}^{-1}\right)$ & Reference \\
\hline K. marxianus NIRE-K3.1 & $28.91 \pm 0.2$ & $15.7 \pm 0.4$ & $4.67 \pm 0.33$ & This study \\
\hline K. marxianus NIRE-K1.1 & $27.43 \pm 2.20$ & $18.75 \pm 0.9$ & $2.88 \pm 0.21$ & Sharma et al. (2016) \\
\hline Saccharomyces cerevisiae YSX3-TAL1M & 19.86 & 6.15 & - & Jin et al. (2005) \\
\hline S. cerevisiae TMB3130 & 17.9 & 5.54 & 5.19 & Garcia Sanchez et al. (2010) \\
\hline S. cerevisiae H131-A3-AL ${ }^{\mathrm{CS}}$ & 30 & - & 12.3 & Zhou et al. (2012) \\
\hline S. cerevisiae SR8u & 40 & - & 15.6 & Li et al. (2016) \\
\hline S. cerevisiae SR7e3 & 80 & - & 29.6 & Kim et al. (2013) \\
\hline Spathaspora passalidarum UFMG-CM-Y469 & $40-50$ & $1.1 \pm 0.0$ & $20.2 \pm 0.1$ & Cadete et al. (2016) \\
\hline Sp. passalidarum CBS 10155T & $40-50$ & $0.6 \pm 0.0$ & $20.3 \pm 0.2$ & Cadete et al. (2016) \\
\hline Sp. roraimanensis UFMG-CM-Y477T & $40-50$ & $22.7 \pm 0.3$ & $5.8 \pm 0.3$ & Cadete et al. (2016) \\
\hline S. cerevisiae SXA-R2P-E & 40 & - & 18 & Lee et al. (2014) \\
\hline S. cerevisiae H131-A3-CS & 40 & - & 17.2 & Zhou et al. (2012) \\
\hline S. cerevisiae SR8 & 40 & - & 15.6 & Wei et al. (2013) \\
\hline S. cerevisiae CEN.PK2-1C-TMB 3424 & 60 & - & 21.06 & Runquist et al. (2010) \\
\hline
\end{tabular}

vanillin, syringaldehyde, and hydroxybenzoic acid have also been developed in ethanologenic yeast by approaching adaptation strategy (Landaeta et al., 2013; Slininger et al., 2015). In another study, adaptation of S. stipitis was performed in acid hydrolyzate and evolved strain showed enhanced tolerance with improved fermentation of hydrolyzate in shorter duration (Nigam, 2001). Similarly, the evolutionary engineering of S. stipitis has been done in hardwood spent sulfite liquor and various evolved strains with improved tolerance to inhibitor present in liquor have been obtained (Pereira et al., 2015). One of the evolved strain was able to tolerate $60 \%(\mathrm{v} / \mathrm{v})$ liquor concentration with higher substrate uptake rate $\left(0.22 \mathrm{~g} \mathrm{l}^{-1} \mathrm{~h}^{-1}\right)$, ethanol efficiency, and ethanol yield $\left(0.16 \mathrm{~g} \mathrm{~g}^{-1}\right)$ (Pereira et al., 2015). Furthermore, Table 3 summarizes to compare xylose utilization, and ethanol and xylitol production of evolved strain K. marxianus NIRE-K3.1 with different reported strains. The previously mentioned illustrations signify the success of adaption, and its importance to develop a process for production of ethanol and other value-added products from lignocellulosic biomass.

\section{CONCLUSION}

Sustainable development of economic bioethanol production is now a necessity of society. Unfortunately, there is no single strain available for industrial production of bioethanol by coutilizing xylose and glucose. Moreover, the cost of production may also be decreased through the production of value-added products. On the other hand, tolerance to some inhibitors including HMF and furfural produced during pretreatment of biomass should also be developed. As reported in this study, the evolutionary adaptation has been proven very useful strategy to develop an industrial important strain. The developed strain $K$. marxianus NIRE-K3.1 showed significant improvement including shorter in lag phase, improved xylitol and ethanol yields, and enhanced xylose uptake rate, which may contribute in the economical production of ethanol and value-added products from lignocellulosic residues.

\section{AUTHOR CONTRIBUTIONS}

NS performed the experiments and wrote the manuscript. SB and RA helped in designing the experiments. SK supervised and edited the manuscript.

\section{ACKNOWLEDGMENTS}

One of the authors (NS) is thankful to Sardar Swaran Singh National Institute of Bio-Energy, Kapurthala for providing Senior Research Fellowship and I. K. Gujral Punjab Technical University, Kapurthala for providing Ph.D. registration (Reg. No. 1422002). Authors also gratefully acknowledge the Ministry of New and Renewable Energy, Govt. of India for providing financial supports to carry out this research.

\section{REFERENCES}

Agbogbo, F. K., Haagensen, F. D., Milam, D., and Wenger, K. S. (2008). Fermentation of acid pretreated corn stover to ethanol without detoxification using Pichia stipitis. Appl. Biochem. Biotechnol. 145, 53-58. doi:10.1007/s12010-007-8056-4

Apel, A. R., Ouellet, M., Szmidt-Middleton, H., Keasling, J. D., and Mukhopadhyay, A. (2016). Evolved hexose transporter enhances xylose uptake and glucose/xylose co-utilization in Saccharomyces cerevisiae. Sci. Rep. 6, 19512. doi:10.1038/ srep 19512

Arora, R., Behera, S., Sharma, N. K., and Kumar, S. (2015). A new search for thermotolerant yeasts, its characterization and optimization using response surface methodology for ethanol production. Front. Microbiol. 6:889. doi:10.3389/fmicb.2015.00889

Arora, R., Behera, S., Sharma, N. K., and Kumar, S. (2017). Augmentation of ethanol production through statistically designed growth and fermentation medium using novel thermotolerant yeast isolates. Renew. Energy 109, 406-421. doi:10.1016/j.renene.2017.03.059

Behera, S., Sharma, N. K., Arora, R., and Kumar, S. (2016). Effect of evolutionary adaption on xylosidase activity in thermotolerant yeast isolates Kluyveromyces marxianus NIRE-K1 and NIRE-K3. Appl. Biochem. Biotechnol. 179, 1143-1154. doi:10.1007/s12010-016-2055-2

Cadete, R. M., de Las Heras, A. M., Sandström, A. G., Ferreira, C., Gírio, F., GorwaGrauslund, M. F., et al. (2016). Exploring xylose metabolism in Spathaspora species: XYL1.2 from Spathaspora passalidarum as the key for efficient anaerobic xylose fermentation in metabolic engineered Saccharomyces cerevisiae. Biotechnol. Biofuels 9, 167. doi:10.1186/s13068-016-0570-6 
Chandel, A. K., Chandrasekhar, G., Radhika, K., Ravinder, R., and Ravindra, P. (2011). Bioconversion of pentose sugars into ethanol: a review and future directions. Biotechnol. Mol. Biol. Rev. 6, 8-20. doi:10.5897/BMBR

Chandel, A. K., and Singh, O. V. (2011). Weedy lignocellulosic feedstock and microbial metabolic engineering: advancing the generation of 'Biofuel'. Appl. Microbiol. Biotechnol. 89, 1289-1303. doi:10.1007/s00253-010-3057-6

Choudhary, J., Singh, S., and Nain, L. (2017). Bioprospecting thermotolerant ethanologenic yeasts for simultaneous saccharification and fermentation from diverse environments. Electron. J. Biotechnol. 21, 82-92. doi:10.1016/ j.jbiosc.2016.10.007

Diao, L., Liu, Y., Qian, F., Yang, J., Jiang, Y., and Yang, S. (2013). Construction of fast xylose-fermenting yeast based on industrial ethanol-producing diploid Saccharomyces cerevisiae by rational design and adaptive evolution. BMC Biotechnol. 13:110. doi:10.1186/1472-6750-13-110

Garcia Sanchez, R., Karhumaa, K., Fonseca, C., Sanchez Nogue, V., Almeida, J. R., Larsson, C. U., et al. (2010). Improved xylose and arabinose utilization by an industrial recombinant Saccharomyces cerevisiae strain using evolutionary engineering. Biotechnol. Biofuels 3, 13. doi:10.1186/1754-6834-3-13

Jin, Y. S., Alper, H., Yang, Y. T., and Stephanopoulos, G. (2005). Improvement of xylose uptake and ethanol production in recombinant Saccharomyces cerevisiae through an inverse metabolic engineering approach. Appl. Environ. Microbiol. 71, 8249-8256. doi:10.1128/AEM.71.12.8249-8256.2005

Jõers, A., and Tenson, T. (2016). Growth resumption from stationary phase reveals memory in Escherichia coli cultures. Sci. Rep. 6, 24055. doi:10.1038/ srep 24055

Kahr, H., Helmberger, S., and Jäger, A. G. (2011). "Yeast adaptation on the substrate straw, in World Renewable Energy Congress-Sweden, 8-13 May, Linköping, Sweden.

Kim, S. R., Skerker, J. M., Kang, W., Lesmana, A., Wei, N., Arkin, A. P., et al. (2013). Rational and evolutionary engineering approaches uncover a small set of genetic changes efficient for rapid xylose fermentation in Saccharomyces cerevisiae. PLoS ONE 8:e57048. doi:10.1371/journal.pone.0057048

Koppram, R., Albers, E., and Olsson, L. (2012). Evolutionary engineering strategies to enhance tolerance of xylose utilizing recombinant yeast to inhibitors derived from spruce biomass. Biotechnol. Biofuels 5, 32. doi:10.1186/ 1754-6834-5-32

Kuhad, R. C., Gupta, R., Khasa, Y. P., Singh, A., and Zhang, Y. H. P. (2011). Bioethanol production from pentose sugars: current status and future prospects. Ren. Sus. Energy Rev. 15, 4950-4962. doi:10.1016/j.rser.2011.07.058

Kumar, S., Singh, S. P., Mishra, I. M., and Adhikari, D. K. (2009). Recent advances in production of bioethanol from lignocellulosic biomass. Chem. Eng. Technol. 32, 517-526. doi:10.1002/ceat.200800442

Kumar, S., Singh, S. P., Mishra, I. M., and Adhikari, D. K. (2010). Feasibility of ethanol production with enhanced sugar concentration in bagasse hydrolysate at high temperature using Kluyveromyces sp. IIPE453. Biofuels 1, 697-704. doi: $10.4155 /$ bfs. 10.51

Kuyper, M., Toirkens, M. J., Diderich, J. A., Winkler, A. A., van Dijken, J. P., and Pronk, J. T. (2005). Evolutionary engineering of mixed-sugar utilization by a xylose-fermenting Saccharomyces cerevisiae strain. FEMS Yeast Res. 5, 925-934. doi:10.1016/j.femsyr.2005.04.004

Landaeta, R., Aroca, G., Acevedo, F., Teixeira, J. A., and Mussatto, S. I. (2013). Adaptation of a flocculent Saccharomyces cerevisiae strain to lignocellulosic inhibitors by cell recycle batch fermentation. Appl. Energy 102, 124-130. doi:10.1016/j.apenergy.2012.06.048

Lee, S. M., Jellison, T., and Alper, H. S. (2014). Systematic and evolutionary engineering of a xylose isomerase-based pathway in Saccharomyces cerevisiae for efficient conversion yields. Biotechnol. Biofuels 7, 122. doi:10.1186/ s13068-014-0122-x

Li, X., Par, A., Estrela, R., Kim, S. R., Jin, Y. S., and Cate, J. H. D. (2016). Comparison of xylose fermentation by two high-performance engineered strains of Saccharomyces cerevisiae. Biotechnol. Rep. 9, 53-56. doi:10.1016/j.btre. 2016.01.003

Liu, E., and Hu, Y. (2010). Construction of a xylose-fermenting Saccharomyces cerevisiae strain by combined approaches of genetic engineering, chemical mutagenesis and evolutionary adaptation. Biochem. Eng. J. 48, 204-210. doi:10.1016/j.bej.2009.10.011

Martin, C., Marcet, M., Almazan, O., and Jonsson, L. J. (2007). Adaptation of a recombinant xylose-utilizing Saccharomyces cerevisiae strain to a sugarcane bagasse hydrolysate with high content of fermentation inhibitors. Bioresor. Technol. 98, 1767-1773. doi:10.1016/j.biortech.2006.07.021

Mihoub, F., Mistou, M. Y., Guillot, A., Leveau, J. Y., Boubetra, A., and Billaux, F. (2003). Cold adaptation of Escherichia coli: microbiological and proteomic approaches. Int. J. Food Microbiol. 89, 171-184. doi:10.1016/S01681605(03)00119-3

Mohagheghi, A., Linger, J., Smith, H., Yang, S., Dowe, N., and Pienkos, P. T. (2014). Improving xylose utilization by recombinant Zymomonas mobilis strain b through adaptation using 2-deoxyglucose. Biotechnol. Biofuels 7, 19. doi:10.1186/1754-6834-7-19

New, A. M., Cerulus, B., Govers, S. K., Perez-Samper, G., Zhu, B., Boogmans, S., et al. (2014). Different levels of catabolite repression optimize growth in stable and variable environments. PLoS Biol. 12:e1001764. doi:10.1371/journal. pbio. 1001764

Nigam, J. N. (2001). Development of xylose-fermenting yeast Pichia stipitis for ethanol production through adaptation on hardwood hemicellulose acid prehydrolysate. J. Appl. Microbiol. 90, 208-215. doi:10.1046/j.1365-2672.2001.01234.x

Pereira, S. R., Sanchez, I., Nogue, V., Frazao, C. J., Serafim, L. S., GorwaGrauslund, M. F., et al. (2015). Adaptation of Scheffersomyces stipitis to hardwood spent sulfite liquor by evolutionary engineering. Biotechnol. Biofuels 8, 50. doi:10.1186/s13068-015-0234-y

Qi, X., Zha, J., Liu, G. G., Zhang, W., Li, B. Z., and Yuan, Y. J. (2015). Heterologous xylose isomerase pathway and evolutionary engineering improve xylose utilization in Saccharomyces cerevisiae. Front. Microbiol. 6:1165. doi:10.3389/ fmicb.2015.01165

Rodrussamee, N., Lertwattanasakul, N., Hirata, K., Suprayogi, Limtong, S., Kosaka, T., et al. (2011). Growth and ethanol fermentation ability on hexose and pentose sugars and glucose effect under various conditions in thermotolerant yeast Kluyveromyces marxianus. Appl. Microbiol. Biotechnol. 90, 1573-1586. doi:10.1007/s00253-011-3218-2

Runquist, D., Hahn-Hagerdal, B., and Bettiga, M. (2010). Increased ethanol productivity in xylose-utilizing Saccharomyces cerevisiae via a randomly mutagenized xylose reductase. Appl. Environ. Microbiol. 76, 7796-7802. doi:10.1128/ AEM.01505-10

Sharma, N. K., Behera, S., Arora, R., and Kumar, S. (2014). "Genetic modification in yeast for simultaneous utilization of glucose and xylose," in Recent Advances in Bioenergy Research, Vol. III, eds S. Kumar, A. K. Sarma, S. K. Tyagi, and Y. K. Yadav (Kapurthala: SSS-NIRE), 194-207.

Sharma, N. K., Behera, S., Arora, R., and Kumar, S. (2016). Enhancement in xylose utilization using Kluyveromyces marxianus NIRE-K1 through evolutionary adaptation approach. Bioprocess Biosyst. Eng. 39, 835-843. doi:10.1007/ s00449-016-1563-3

Shen, Y., Chen, X., Peng, B., Chen, L., Hou, J., and Bao, X. (2012). An efficient xylose-fermenting recombinant Saccharomyces cerevisiae strain obtained through adaptive evolution and its global transcription profile. Appl. Microbiol. Biotechnol. 96, 1079-1091. doi:10.1007/s00253-012-4418-0

Silva, C. J., and Roberto, I. C. (2001). Improvement of xylitol production by Candida guilliermondii FTI 20037 previously adapted to rice straw hemicellulosic hydrolysate. Lett. Appl. Microbiol. 32, 248-252. doi:10.1046/j.1472765X.2001.00899.x

Slininger, P. J., Shea-Andersh, M. A., Thompson, S. R., Dien, B. S., Kurtzman, C. P., Balan, V., et al. (2015). Evolved strains of Scheffersomyces stipitis achieving high ethanol productivity on acid- and base-pretreated biomass hydrolyzate at high solids loading. Biotechnol. Biofuels 8, 60. doi:10.1186/s13068-015-0239-6

Wei, N., Quarterman, J., Kim, S. R., Cate, J. H. D., and Jin, Y.-S. (2013). Enhanced biofuel production through coupled acetic acid and xylose consumption by engineered yeast. Nat. Commun. 4, 2580. doi:10.1038/ncomms3580

Wilkins, M. R., Mueller, M., Eichling, S., and Banat, I. M. (2008). Fermentation of xylose by the thermotolerant yeast strains Kluyveromyces marxianus IMB2, IMB4, and IMB5 under anaerobic conditions. Process Biochem. 43, 346-350. doi:10.1016/j.procbio.2007.12.011

Zhang, B., Li, L., Zhang, J., Gao, X., Wang, D., and Hong, J. (2013). Improving ethanol and xylitol fermentation at elevated temperature through substitution of xylose reductase in Kluyveromyces marxianus. J. Ind. Microbiol. Biotechnol. 40, 305-316. doi:10.1007/s10295-013-1230-5

Zhang, J., Zhang, B., Wang, D., Gao, X., Sun, L., and Hong, J. (2015). Rapid ethanol production at elevated temperatures by engineered thermotolerant Kluyveromyces marxianus via the $\mathrm{NADP}(\mathrm{H})$-preferring xylose 
reductase-xylitol dehydrogenase pathway. Metab. Eng. 31, 140-152. doi:10.1016/ j.ymben.2015.07.008

Zhou, H., Cheng, J. S., Wang, B. L., Fink, G. R., and Stephanopoulos, G. (2012). Xylose isomerase overexpression along with engineering of the pentose phosphate pathway and evolutionary engineering enable rapid xylose utilization and ethanol production by Saccharomyces cerevisiae. Metab. Eng. 14, 611-622. doi:10.1016/j.ymben.2012.07.011

Zhu, J. J., Yong, Q., Xu, Y., Chen, S. X., and Yu, S. Y. (2009). Adaptation fermentation of Pichia stipitis and combination detoxification on steam exploded lignocellulosic prehydrolyzate. Nat. Sci. 1, 47-54. doi:10.4236/ns.2009.11009
Conflict of Interest Statement: The authors declare that the research was conducted in the absence of any commercial or financial relationships that could be construed as a potential conflict of interest.

Copyright $\odot 2017$ Sharma, Behera, Arora and Kumar. This is an open-access article distributed under the terms of the Creative Commons Attribution License (CC BY). The use, distribution or reproduction in other forums is permitted, provided the original author(s) or licensor are credited and that the original publication in this journal is cited, in accordance with accepted academic practice. No use, distribution or reproduction is permitted which does not comply with these terms. 